\title{
Phasic and Tonic Smooth Muscle Function of the Partially Obstructed Guinea Pig Intestine
}

\author{
Jingbo Zhao, ${ }^{1,2}$ Donghua Liao, ${ }^{1,2}$ Jian Yang, ${ }^{1}$ and Hans Gregersen ${ }^{3}$ \\ ${ }^{1}$ Mech-Sense, Aalborg Hospital, 9000 Aalborg, Denmark \\ ${ }^{2}$ Institute of Clinical Medicine, Aarhus University Hospital, 8000 Aarhus, Denmark \\ ${ }^{3}$ Sino-Danish Center for Education and Research, Aarhus University, 8000 Aarhus, Denmark
}

Correspondence should be addressed to Jingbo Zhao, jz@rn.dk

Received 28 June 2011; Accepted 29 August 2011

Academic Editor: Henk Granzier

Copyright (C) 2011 Jingbo Zhao et al. This is an open access article distributed under the Creative Commons Attribution License, which permits unrestricted use, distribution, and reproduction in any medium, provided the original work is properly cited.

\begin{abstract}
This study was to generate phasic and tonic stress-strain curves for evaluation of smooth muscle function in the obstructed guinea pig jejunum. Partial and sham obstruction of the jejunum in guinea pigs was created surgically, with guinea pigs not being operated on served as normal controls. The animals survived 2, 4, 7, and 14 days, respectively. The jejunal segment was distended to $10 \mathrm{~cm} \mathrm{H}_{2} \mathrm{O}$. The pressure and outer diameter changes were recorded. Passive conditions were obtained by using papaverine. Total phasic, tonic, and passive circumferential stress and strain were computed from the diameter and pressure data with reference to the zero-stress-state geometry. The active phasic and tonic stresses were defined as the total phasic and tonic stress minus the passive stress. The thickness of intestinal muscle layers increased in a time-dependent manner after obstruction. The amplitude of passive, total phasic, total tonic, active phasic, and active tonic circumferential stresses increased as function of strain 7 days after obstruction. However, when normalized to muscle layer thickness, the amplitude of active stresses did not differ among the groups. In conclusion, the long-term-obstructed intestine exhibits increased total smooth muscle contraction force. However, the contraction force per smooth muscle unit did not increase.
\end{abstract}

\section{Introduction}

Small bowel obstruction is a common clinical problem resulting from congenital [1-3] or acquired causes [4-7]. Small intestinal mechanical obstruction can be reproduced in laboratory animals using rings of different materials. Proximal to the partial obstruction site, the intestinal segment remodels morphologically and biomechanically [814]. Partial obstruction causes intestinal smooth muscle hyperplasia and hypertrophy $[8,9,11,12,15-17]$. Increased contraction force of remodeled smooth muscle layer has been reported, but the force was decreased when normalized to the tissue wet weight [15]. Furthermore, the obstructed intestinal wall became stiffer during longstanding partial obstruction $[11,12,14]$. Stiffening likely affects smooth muscle function. Therefore, it is important to study the function of remodeled intestinal smooth muscle due to partial obstruction.

It is possible to obtain isometric length-tension diagrams of phasic and tonic smooth muscle contraction in vitro [18].
Tools have now been developed for studying the active (phasic and tonic contractions) and passive length-tension behavior in the human gut in vivo using impedance planimetric distension [19-21]. From a biomechanical standpoint, muscle mechanical properties must be described in terms of stress and strain, that is, the force per area and tissue deformation. Computation of the stress depends on the wall thickness which cannot be directly measured in vivo. However, it is possible to measure the wall thickness in vitro and thus obtain the stress-strain relationship of the intestinal wall with reference to the zero-stress state [22]. Furthermore, the passive, tonic, and phasic stress-strain curves can be obtained when using papaverine to abolish smooth muscle activity [22].

In the present study we computed stress-strain data for assessment of the smooth muscle function of partially obstructed intestinal segments from guinea pigs. We hypothesized that the stress-strain properties of the remodeled small intestine change and that the remodeling is determined by the stress similar to the cardiovascular system [23]. 


\section{Materials and Methods}

2.1. Animals and Groups. The protocol was approved by the Danish Committee for Animal Experimentation. Male guinea pigs (600-800 g) were divided into four obstructed and four sham-obstructed groups living for 2, 4, 7, and 14 days, respectively. Ten age-matched guinea pigs not exposed to surgery were used as normal controls. We have long-term experience with the operative procedures $[11,12,14]$, which is likely the reason for a mortality rate below $20 \%$. The final number of animals was 6 in each obstructed group and 4 in each of the sham-obstructed group. The guinea pigs had access to water but were restricted from food intake from the night before the operations and experiments. The animals were weighted daily.

2.2. Creation of Partial Small Intestine Obstruction in Guinea Pig. The surgical procedure for partially obstructing the small intestine is well established $[11,14]$. Atropine (Atropin DAK, Denmark) $0.3 \mathrm{mg} * \mathrm{~kg}^{-1}$ s.c. was given $30 \mathrm{mi}-$ nutes prior to anesthesia with Hypnorm $0.5 \mathrm{mg}$ and Dormicum $0.25 \mathrm{mg}$ per $100 \mathrm{~g}$ body weight (Hypnorm:Dormicum : sterile water $=1: 1: 2$; subcutaneous injection). A small midline laparotomy was done when surgical anesthesia was achieved. A loop of the midjejunum was selected, and the mesenterium was carefully incised close to the intestine to create a small window. Care was taken not to damage adjacent vessels or nerves.

A $3.5 \mathrm{~mm}$ wide polyurethane band was passed through the mesenteric window and closed antimesenterically with a $6-0$ silk suture at a circumferential length about one $\mathrm{mm}$ longer than the outer circumference of the small intestine. Hence, a loose fit around the intestine was obtained without any apparent compression of the tissue. In the shamobstructed group, the mesenteric incision was made and marked with a $6-0$ silk suture but no band was placed. The abdominal wall was closed with $4-0$ silk suture. Buprenorphine (Temgesic, Reckitt \& Colman, UK) $0.05 \mathrm{mg}^{*} \mathrm{~kg}^{-1}$ was given subcutaneously to counter postoperative pain along with $10 \mathrm{~mL}$ saline to prevent dehydration. The animals were inspected and weighed daily after the operation. Animals in poor clinical condition were euthanized and excluded from the study.

2.3. In Vitro Intestinal Preparation. The guinea pigs were anesthetized with Hypnorm and Dormicum when the scheduled time had arrived. The abdominal cavity was opened and the intestine carefully dissected. A ten $\mathrm{cm}$ intestinal segment proximal to the band in the obstructed animals and segments from the corresponding location in shamobstructed and normal animals were removed. A $0.5 \mathrm{~cm}$ long intestinal piece from the proximal end of the excised segment was cut and used for histological analysis. Further two short ring-shaped pieces perpendicular to the longitudinal axis were cut and used for zero-stress state analysis. The remaining segment was immediately put into the organ bath containing Krebs solution of the following composition $\left(\mathrm{mmol} * \mathrm{~L}^{-1}\right): \mathrm{NaCl}, 118 ; \mathrm{KCl}, 4.7 ; \mathrm{NaHCO}_{3}, 25 ; \mathrm{NaH}_{2} \mathrm{PO}_{4}$, 1.0; $\mathrm{MgCl}, 1.2 ; \mathrm{CaCl}_{2}-\mathrm{H}_{2} \mathrm{O}, 2.5$; glucose, 11 ; ascorbic acid,
0.11. The $37^{\circ} \mathrm{C}$ Krebs solution was aerated with a gas mixture $\left(95 \% \mathrm{O}_{2}\right.$ and $\left.5 \% \mathrm{CO}_{2}, \mathrm{pH} 7.4\right)$. Thirty minutes equilibrating time was needed for recovery of the intestinal motility.

2.4. Ramp Distension Experimental Setup (Figure 1). The in vitro initial length of the intestinal segment was measured. The proximal end of the intestinal segment was tied on the cannula with silk threads. The cannula was via a tube connected to a syringe containing Krebs solution for applying luminal pressures $\left(0.8 \mathrm{~mL} * \mathrm{~min}^{-1}\right)$. The lumen was pressurized by a pump (Genie Programmable Syringe Pump, World Precision Instrument, Stevenage, UK). The distal end of the intestinal segment was tied by a silk thread on the three-way tube connected to a micromanipulator that could stretch the intestinal segment in longitudinal direction. The ramp distension experiment with pressure up to $10.0 \mathrm{~cm} \mathrm{H}_{2} \mathrm{O}$ was done on the intestinal segment at the longitudinal stretch ratios $0 \%, 10 \%$, and $20 \%$. The pressure probe was inserted into the intestinal lumen through the cannula. The pressure was measured at three locations with $0.5 \mathrm{~cm}$ intervals. The location of the most proximal pressure hole was marked on the intestinal surface. The intestinal diameters at the locations where the pressure was recorded in the intestinal segments were videotaped by a CCD camera (Sony, Japan) on a stereomicroscope. The data sampling frequency for pressure and diameter data was 10/second.

2.5. The Zero-Stress State of the Intestinal Segment. The method for determination of the gastrointestinal zero-stress state has been described in detail previously [24, 25]. Onetwo $\mathrm{mm}$ wide rings were transferred to a calcium-free Krebs solution with EGTA and papaverine. This represented the no-load state that was photographed. Then, each ringshaped segment was cut radially under the microscope resulting in an open sector geometry. Photographs representing the zero-stress state were taken $\sim 60$ min after the radial cutting to allow viscoelastic creep to occur.

2.6. Histological Analysis of the Small Intestine. The segment was fixed in $10 \%$ buffered formalin over 24 hours followed by dehydration in a series of graded ethanol $(70 \%, 96 \%$, and 99\%) and embedding in paraffin. Five-micron sections were cut perpendicular to the mucosa surface, and the paraffin was cleared from the slides with coconut oil (over $15 \mathrm{~min} .60^{\circ} \mathrm{C}$ ). Redehydration occurred in 99\%, 96\%, and 70\% ethanol followed by staining with hematoxylin and eosin. The layer thickness was measured by the same pathologist in a blinded review. Twelve determinations were made on each specimen and averaged.

2.7. Mechanical Data Analysis. Calculation was the assessment of the no-load state, zero-stress state dimensions and the outer diameters of the specimen at varying pressures. The Kirchhoff stress and Green's strain in the intestinal wall at a given pressure were computed assuming circular geometry as follows: 


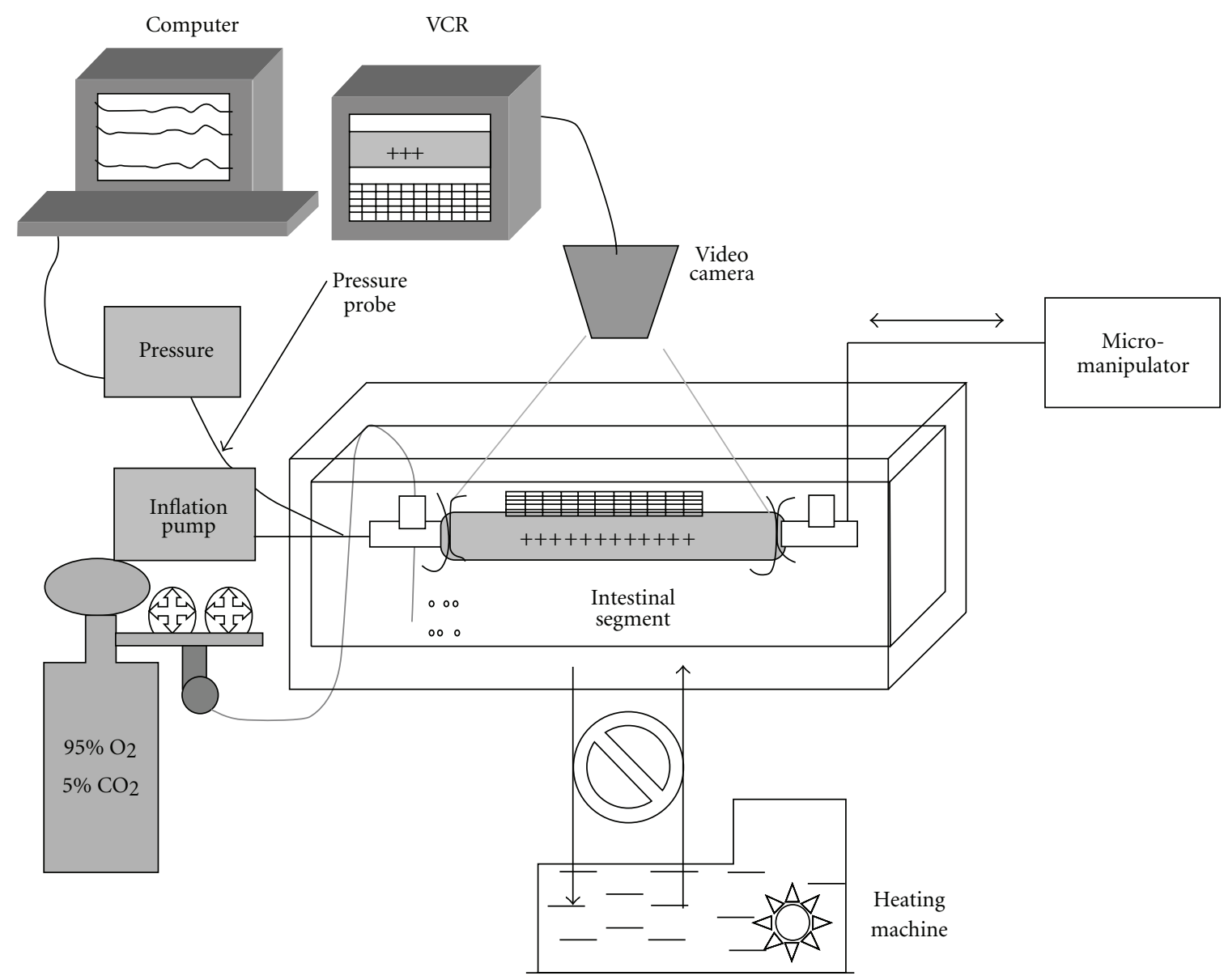

FIGURE 1: Experimental setup. The organ bath is composed of an inside chamber and an outside chamber. The Krebs solution contained in the small chamber was maintained constant at $37^{\circ} \mathrm{C}$ by circulating hot water in the big chamber using a heating machine. The intestinal segment was placed in the small organ bath in the Krebs solution. The intestinal distension was applied by a pump and the longitudinal length was set by a micromanipulator. The three-channel pressure probe was used to measure the pressures. The diameter changes of the intestinal segments were videotaped through a stereomicroscope.

\section{Circumferential Kirchhoff's stress:}

$$
S_{\theta}=\frac{\Delta P r_{i-p}}{h_{p} \lambda_{\theta}^{2}}
$$

Circumferential midwall Green's strain:

$$
E_{\theta}=\frac{\lambda_{\theta}^{2}-1}{2}
$$

where $\Delta P$ is the transmural pressure difference, $r$ is the luminal radius $h$ is the wall thickness and $\lambda_{\theta}$ is the circumferential stretch ratio.

Stress and strain data immediately before the contraction (stress and strain thresholds) and at maximal contraction (maximal amplitude of stress and strain) were used for further analysis.

The total phasic stress and the total tonic stresses (both composed of active and passive tissue properties) were extracted from the top points during contraction and the baseline between the contractions during the distension (Figure 2(a)). The passive stress was extracted from the data obtained during distension after administration of papaverine. The strain points were not fixed; hence, it was not possible to directly compare between different samples and groups. Consequently curve fitting was applied.

The total tonic and passive stresses increased in an exponential-like way as function of strain. Consequently the stress-strain curves were fitted to the exponential function equation

$$
S=\left(S^{*}+\beta\right) e^{\alpha\left(E-E^{*}\right)}-\beta,
$$

where $S^{*}$ and $E^{*}$ are the stress and strain at a physiological reference level [25].

The total phasic circumferential stress-strain curves increased in a polynomial way as function of strain. As a consequence the stress-strain curves were fitted to the polynomial equation

$$
S=S_{0}+a_{1} E^{3}+a_{2} E^{2}+a_{3} E^{1},
$$

where $a_{1}, a_{2}$, and $a_{3}$ are constants. 


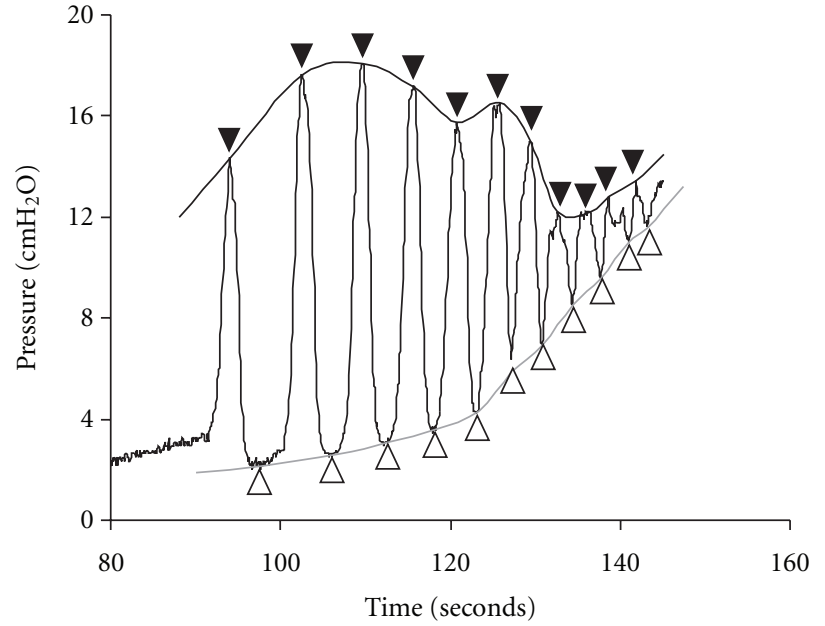

(a)

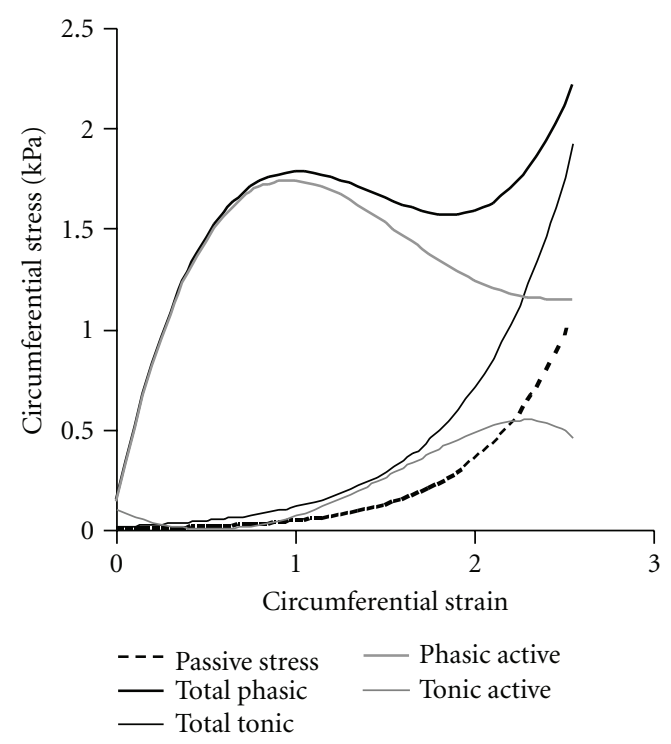

(b)

FIgURE 2: (a) illustrates maximum amplitudes during contraction and the baseline between the contractions during distension. The closed symbols above the curve mark the phasic part (black line). The open symbols under the curve mark the tonic part (gray line). The pressures from the phasic and tonic parts were used to compute the total phasic and tonic stress. (b) illustrates an example of passive, total phasic, total tonic, phasic active, and tonic active stresses as function of strains from a normal animal. The passive and the total tonic stress increased exponentially as function of strain, whereas the total phasic stress increased in a polynomial way. The phasic and tonic active stresses were obtained by the total phasic and tonic stresses minus the passive stress.

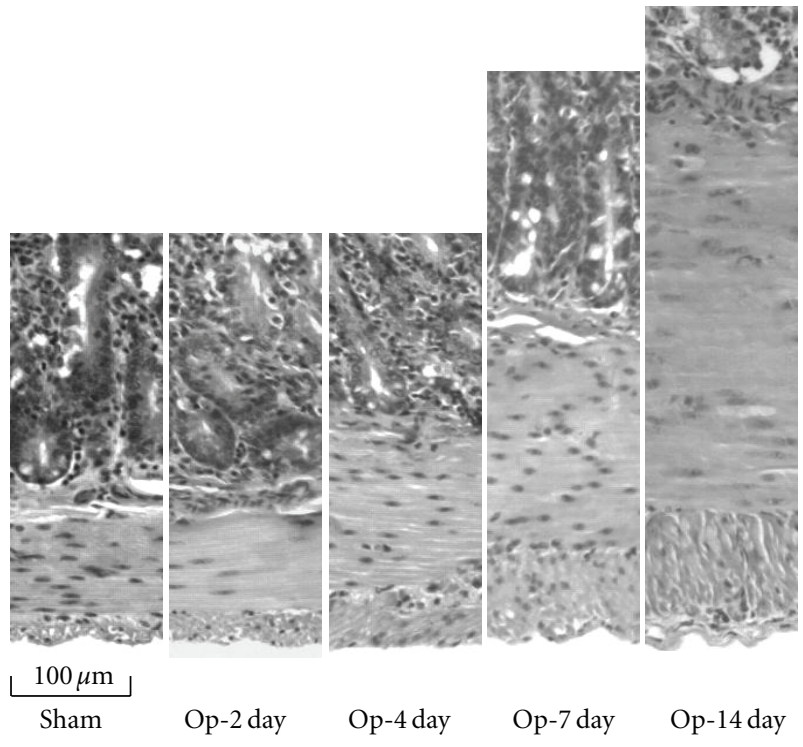

FIGURE 3: The typical pattern of the muscle layer proliferation. The hyperplasia of muscle layer was noted after two days of postobstruction. Seven days after obstruction, the hypertrophy of both muscle layers was also observed. The circumferential muscle layer increased much more than the longitudinal muscle layer.

The active phasic and tonic stresses were defined as the total phasic and tonic stresses minus the passive stress (Figure 2(b))

active phasic stress $=$ total phasic stress - passive stress, active tonic stress $=$ total tonic stress - passive stress.
The active phasic and tonic stresses were normalized to muscle layer thickness as follows:

$$
\begin{gathered}
\text { normalized active phasic stress }=\frac{\text { active phasic stress }}{\text { muscle thickness }}, \\
\text { normalized active tonic stress }=\frac{\text { active tonic stress }}{\text { muscle thickness }} .
\end{gathered}
$$

The muscle layer thickness (um) was obtained by histological measurement.

2.8. Statistical Analysis. The results were expressed as means \pm SEM unless indicated otherwise. The total phasic, total tonic, active phasic, and active tonic stresses were compared between different groups by ANOVA analysis. The normalized active phasic and tonic stresses as function of strain were also compared between groups. The results were regarded as significant when $P<0.05$.

\section{Results}

3.1. General Data. The peritoneum had no signs of inflammation or adhesions that could potentially influence the mechanical properties and intestinal contraction.

The intestinal segments from the animals obstructed for 2 days and 4 days were clearly dilated. After seven days, intestinal hypertrophy was also visible. No changes were observed in the sham-obstructed and normal groups. Histological analysis showed that the thickness of both the submucosa layer and muscle layer increased during the development 


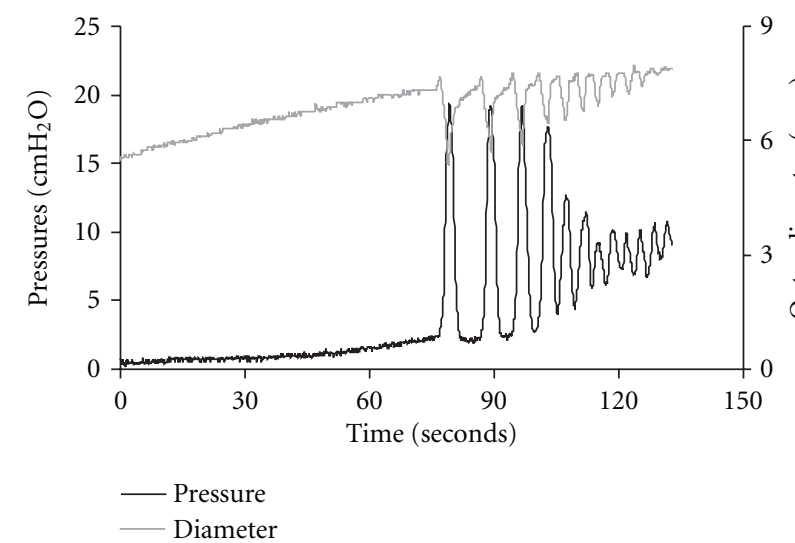

(a)

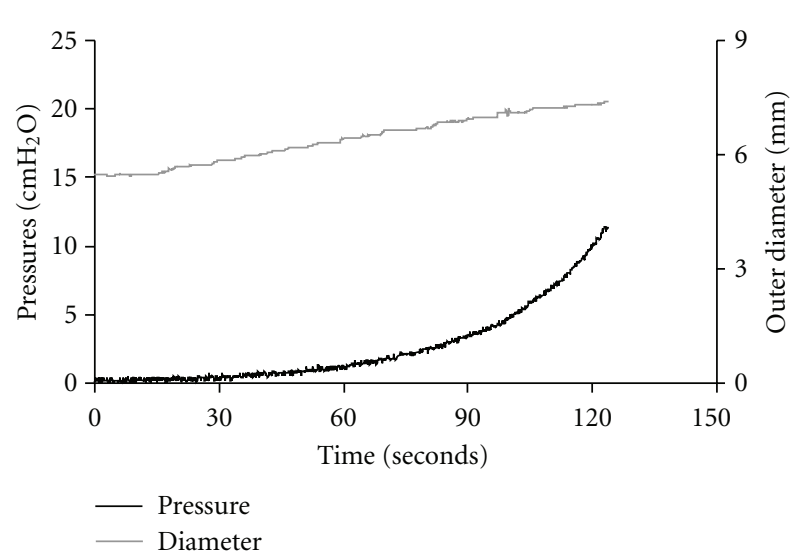

(b)

FIGURE 4: Illustration of ramp distension curves of the pressure and diameter of jejunal segment without longitudinal stretch obtained from a normal control animal. Waves of peristaltic contraction were clearly observed (a). The smooth muscle contraction was abolished by papaverine (b).

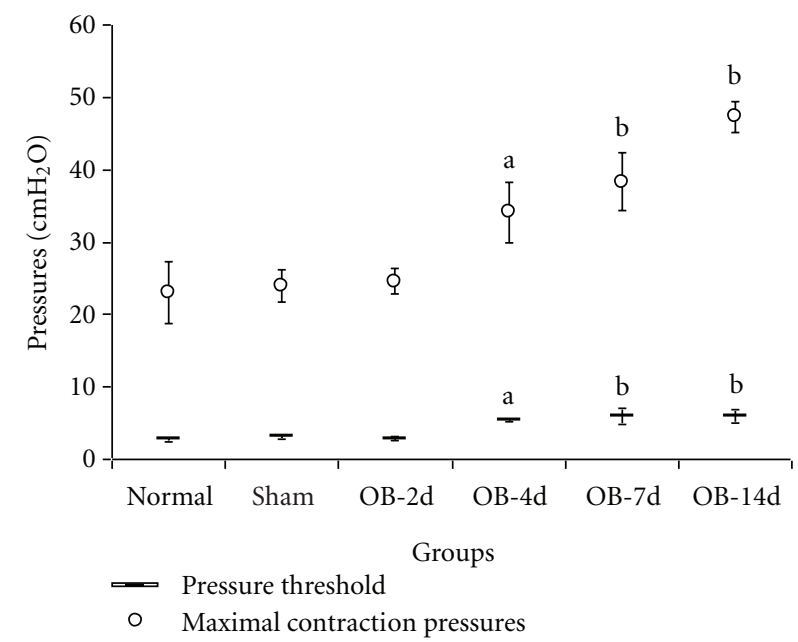

(a)

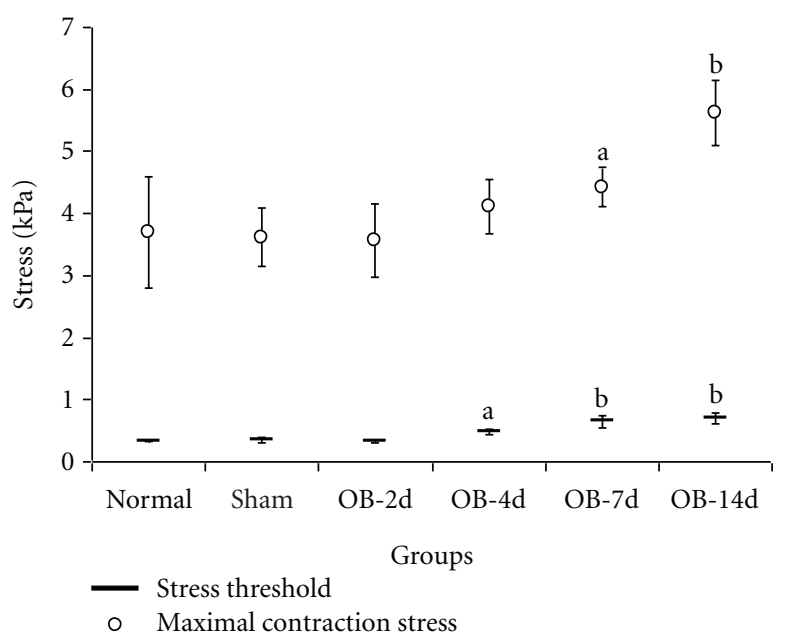

(b)

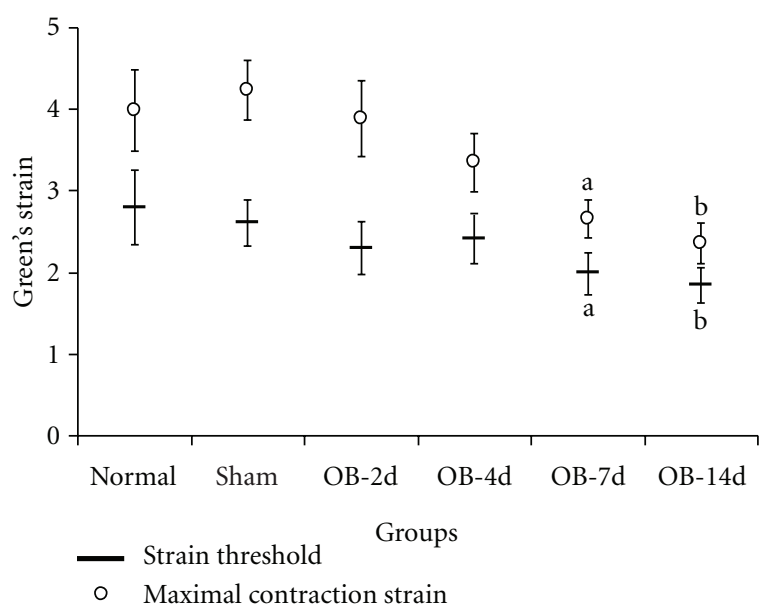

(c)

Figure 5: Illustration of the pressures (a), stresses (b), and strains (c) at the contraction threshold and at the maximum contraction in the groups. Threshold and maximal amplitude of pressures and stresses increased, and strain decreased as function of the obstruction period during ramp distension. Significant differences were found after 4 days of obstruction compared with normal and sham-obstructed controls $\left({ }^{\mathrm{a}} P<0.05,{ }^{\mathrm{b}} P<0.01\right)$. 

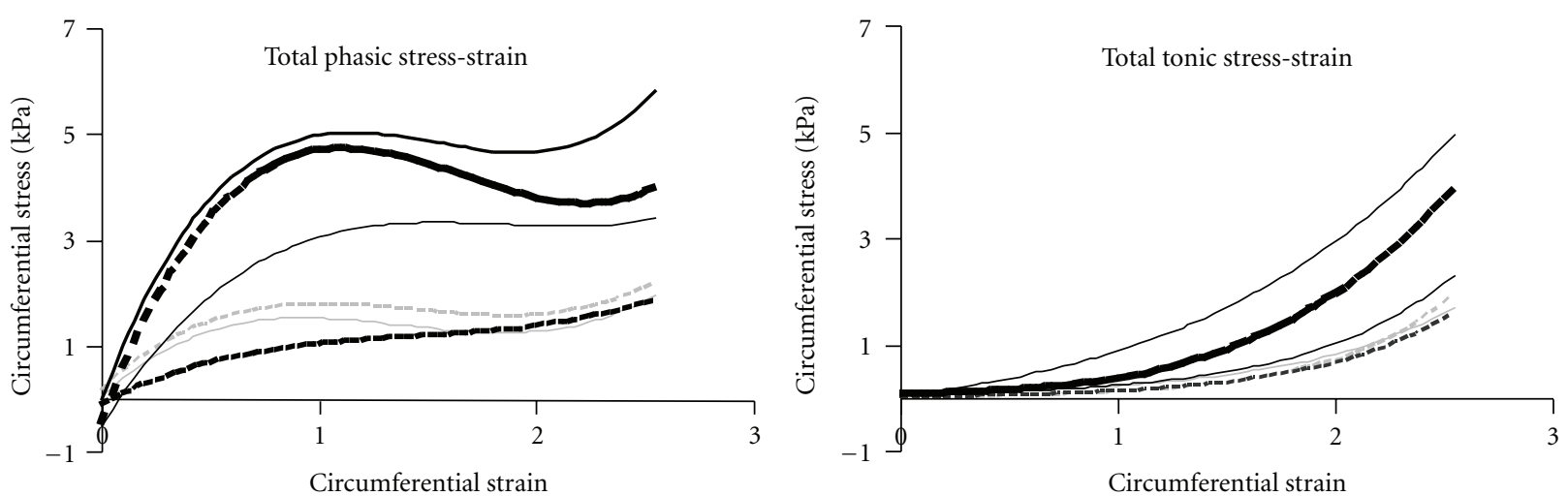

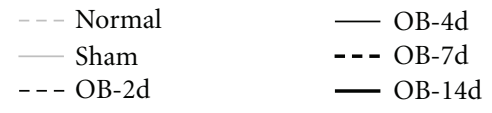

(a)
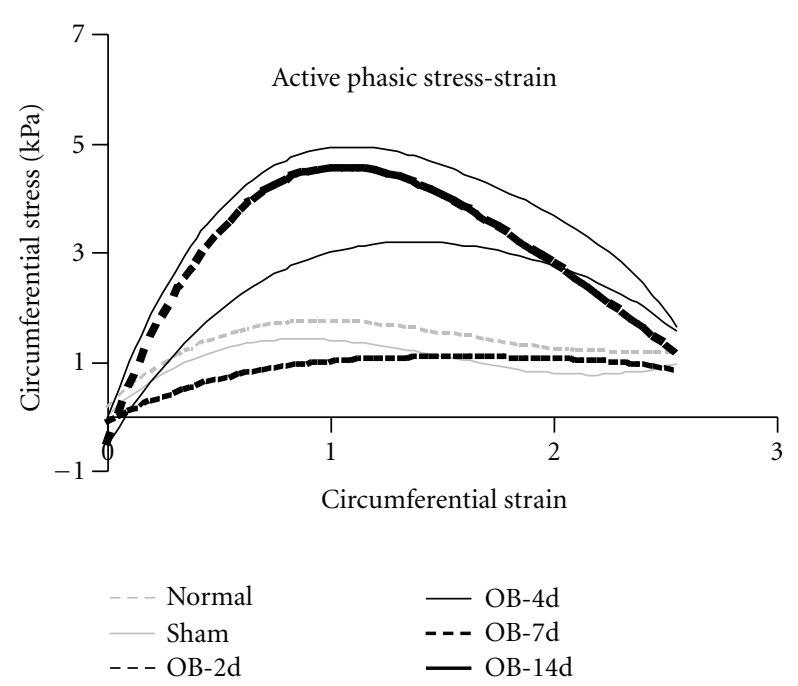

(c)

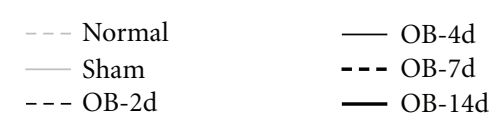

(b)

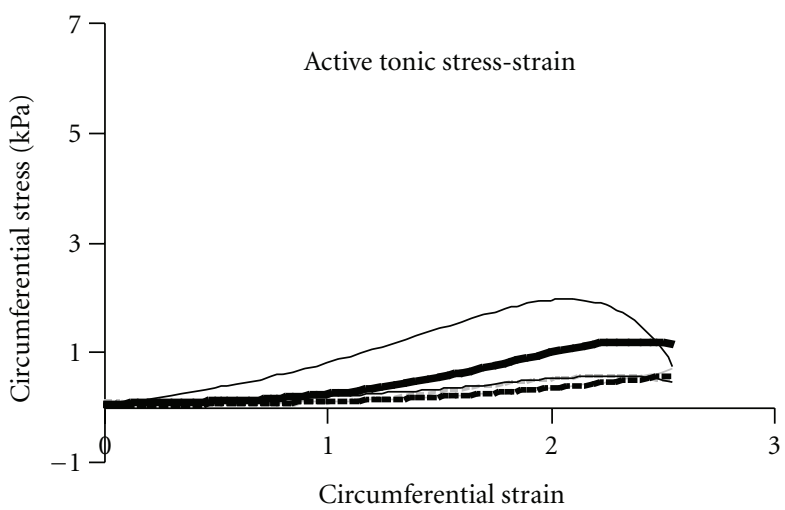

--- Normal
- Sham
--- OB-2d

(d)

Figure 6: The total phasic and tonic stresses as function of strains are presented at the top panel and the computed active phasic and tonic stresses as function of strain at the bottom. The amplitude of total phasic, total tonic, active phasic, and active tonic circumferential stresses increased after 7 days obstruction compared with normal and sham controls $(P<0.05$ and 0.01$)$. Furthermore, the maximum for the active phasic and active tonic stresses differed.

of the obstruction (Table 1). Muscle layer thickening was observed after 4 days of obstruction whereas submucosal thickening was observed after 7 days of obstruction. The muscle hyperplasia was observed two days postobstruction. Seven days postobstruction the intestinal segments were also visibly hypertrophied. A typical pattern of the muscle layer proliferation is shown in Figure 3. The circumferential muscle layer increased much more than the longitudinal muscle layer. After 7 days of obstruction, hypertrophy is evident in both muscle layers.

3.2. Pressure-Diameter Curves. Figure 4 shows the pressure and diameter changes during the distension from a normal jejunal segment without longitudinal stretch applied. Waves of peristaltic contraction were clearly observed both from pressure and diameter curves (Figure 4(a)). The pressure increased whereas the diameter decreased at each contraction wave. Peristaltic contractions were not observed when using papaverine (Figure 4(b)).

The pressures, stresses, and strains at the contraction threshold and maximal contraction points in different groups are shown in Figure 5. During ramp distension-induced contractions, the threshold and maximal pressure amplitude (Figure 5(a)) and stresses (Figure 5(b)) increased after obstruction. However, the strains at the contraction threshold and maximal contraction (Figure 5(c)) decreased during the development of the obstruction. Significant differences were found after 4 days of obstruction compared with normal and sham-obstructed controls $(P<0.05$, $P<0.01)$. Thus the remodeled intestine due to obstruc- 


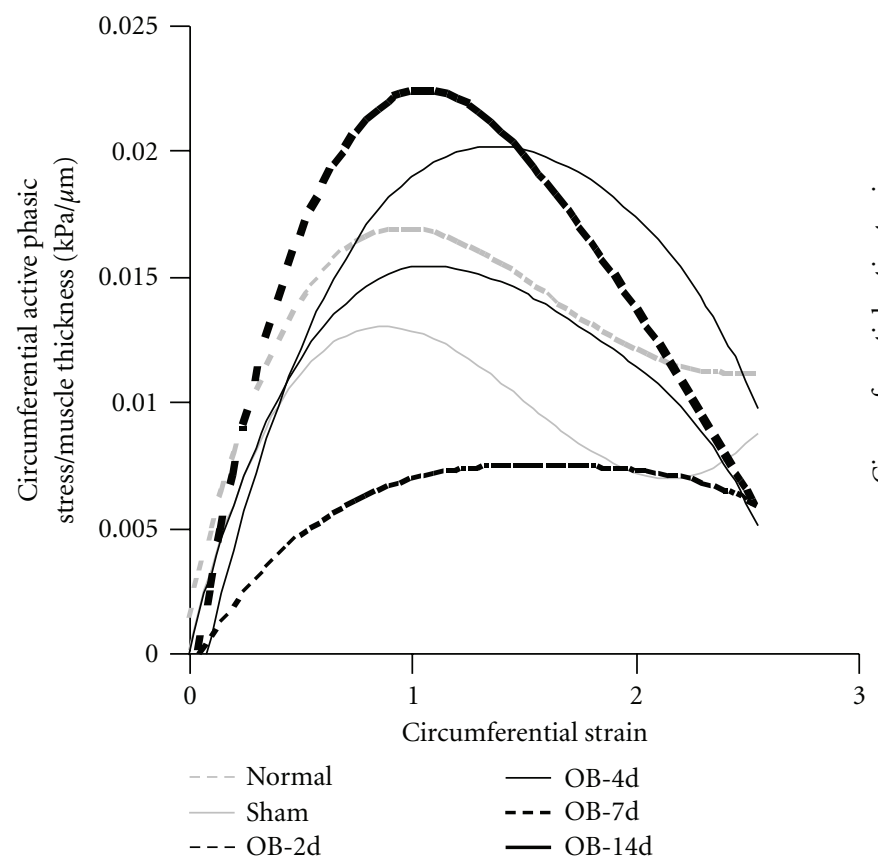

(a)

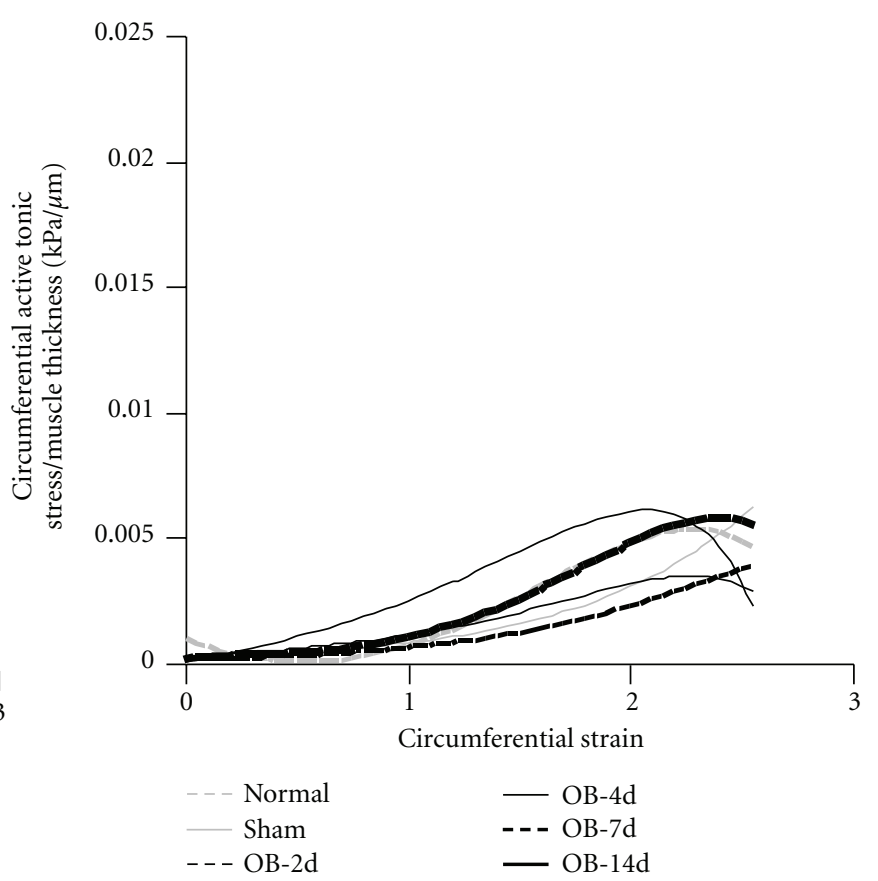

(b)

FIGURE 7: The normalized active phasic and tonic stresses illustrated as function of strains. When normalized to muscle layer thickness, the amplitude of active phasic and tonic stresses did not differ among the groups $(P>0.05)$.

TABLE 1: Layer thickness related to duration of obstruction $(\mu \mathrm{m})$.

\begin{tabular}{lcccc}
\hline Groups & Mucosa & Submucosa & Circumferential muscle & Longitudinal muscle \\
\hline Normal & $861.1 \pm 134.3$ & $42.9 \pm 11.2$ & $79.3 \pm 24.1$ & $36.2 \pm 7.5$ \\
Sham-obstructed & $816.2 \pm 118.3$ & $35.5 \pm 12.6$ & $90.4 \pm 15.1$ & $37.8 \pm 8.8$ \\
Obstructed for 2 days & $905.3 \pm 109.9$ & $45.3 \pm 11.8$ & $107.3 \pm 31.4$ & $37.4 \pm 9.5$ \\
Obstructed for 4 days & $1001.1 \pm 135.9$ & $34.5 \pm 8.5$ & $116.5 \pm 42.3^{*}$ & $42.3 \pm 15.3^{*}$ \\
Obstructed for 7 days & $992.7 \pm 91.6$ & $55.7 \pm 9.9^{*}$ & $145.5 \pm 57.9^{*}$ & $57.9 \pm 10.6^{*}$ \\
Obstructed for 14 days & $1028.1 \pm 167.2$ & $71.8 \pm 18.7^{* *}$ & $240.2 \pm 80.7^{* *}$ & $80.7 \pm 28.9^{* *}$ \\
\hline
\end{tabular}

${ }^{*} P<0.05$; ${ }^{* *} P<0.01$ (compared with normal and sham-obstructed groups).

tion required higher pressure and stress levels to induce contractions, and the induced contractions produced high pressures and stresses during ramp distension. However, the deformation of the intestinal wall became smaller indicating wall stiffening.

3.3. Analysis of Tonic and Phasic Stress-Strain Curves. In accordance with our previous study [22], the passive circumferential stress increased when the intestine was stretched longitudinally. Longitudinal stretching caused the active circumferential stress to decrease (data not shown). However, longitudinal stretch did not influence the differences of tonic and phasic stress-strain curves among the groups. The results below were obtained from the segments at longitudinal stretch of $10 \%$.

Figure 6 illustrates the total phasic (a), total tonic (b), active phasic (c), and active tonic (d) stresses as function of strain in the different groups. The total phasic stress increased in a polynomial way as function of strain whereas the total tonic stress increased in an exponential-like way. At corresponding circumferential strains, the amplitude of total phasic, total tonic, active phasic, and active tonic circumferential stresses increased after 7 days of obstruction compared with normal and sham-obstructed controls $(P<$ 0.05 and 0.01 ). However, when normalized to the muscle layer thickness, the amplitude of active phasic and tonic stresses as function of strain did not differ among the groups (Figure 7, $P>0.05$ ).

\section{Discussion}

The length-tension diagrams known from physiological and pharmacological studies of smooth muscle strips in vitro [19, 26] can be reproduced in intact segment of intestine in vitro as shown in the present and previous studies [22]. From a biomechanical standpoint, phasic and tonic stress-strain data are required for evaluation of smooth muscle mechanical function in intact intestinal segments. The main findings in the current study were that the amplitude of total phasic, 
total tonic, active phasic, and active tonic circumferential stresses increased after 7 days of obstruction. However, when normalized to muscle layer thickness, the amplitude of active stresses did not differ among the groups.

The mechanical properties of the small intestine can be divided into properties arising from a "passive" or connective tissue element, an active ("tonic") element, reflecting baseline muscle activity, and an active ("phasic") element, reflecting the effects of distension-induced neuromuscular function. The passive and the active stress-strain curves depend on the wall structure, the wall mechanical properties, and the smooth muscle contractile properties. Longstanding nonoccluding intestinal obstruction results in structural changes with marked dilatation, increased collagen content, and hypertrophy of especially the muscle layer proximal to the obstruction site $[9,27]$. Thus, stress-strain data are important for understanding the mechanical function of remodeled smooth muscle in the partially obstructed intestine. Length-tension diagrams have been derived from the human gastric antrum and duodenum [21, 28], though butylscopolamine may not have abolished all phasic activity. The present and previous [22] in vitro studies produced tonic and phasic stress-strain curves referenced to the passive stress-strain curve because papaverine completely abolished all smooth muscle activity. Computation of the stress depends on the wall thickness which cannot be directly measured in vivo. However it can be accounted for in vitro.

The partial obstruction narrows the intestine which will increase the resistance to flow during bolus passage. To compensate, the smooth muscle will proliferate to increase contraction force to overcome the resistance. Experimental evidence demonstrated that longstanding partial intestinal obstruction results in marked proliferation of the intestinal muscle layers $[8,9,11,12,14-17]$. In the present study the intestinal muscle layer, especially the circumferential muscle layer, thickness markedly increased during obstruction. The muscle layer increased circumferentially more than longitudinally probably due to increased circumferential stress. Previous studies on pressure contraction curves of obstructed intestinal segments have demonstrated that the total contractile ability of remodeled smooth muscle layer increased $[12,29]$. In the present study the pressure curve (Figure 5(a)) confirmed that the maximal contraction pressure increased after obstruction. In agreement with the proliferation of the smooth muscle layer after 4 days of obstruction, the maximal contraction stress significantly increased indicating increased contraction force. Both muscle proliferation and stress increase in a time-dependent manner as function of obstruction time, indicating that muscle remodeling is determined by the stress. This phenomenon is known from the cardiovascular system [23]. However, the maximal contraction strain significantly decreased during the development of obstruction indicating the wall deformation became smaller due to stiffening [14]. Further stress-strain analysis demonstrated that the total phasic, total tonic, active phasic, and active tonic stresses as function of strain also significantly increased after 7 days of obstruction. Therefore, the increased total contraction force is mainly due to smooth muscle proliferation. As a result, increased total contraction force can compensate to push the content through the partial obstruction site. Furthermore, it is worthwhile to notice that the maximum of active phasic and active tonic stress differ. This probably means that they are regulated in different ways.

Despite the fact that the total contraction force increased after obstruction, the contraction ability of single unit smooth muscle was decreased [15]. It is also interesting to notice at the present study that when normalized to muscle layer thickness, the active phasic and tonic stresses as function of strain did not differ between obstruction groups and sham-obstructed and normal groups. Therefore the remodeled smooth muscle cells may be somehow damaged due to long-term partial obstruction. Earlier studies demonstrated that the hypertrophied muscle cells exhibit ultrastructural changes of sarcoplasmic reticulum, gap connections, and cytoplasmic content and decreased ratio of myofilament to intermediate filament [30-32]. The relative decrease in myofilament content suggests a loss of contractile machinery in remodeled smooth muscle cells due to partial obstruction. Furthermore, it was reported that the stiffness of collagen fibrils could influence vascular smooth muscle cell phenotype and function [33]. The extracellular matrix including collagen was reported to increase due to the obstruction $[11,12]$. We found that the submucosal thickness increased after intestinal obstruction. Therefore, the altered extracellular matrix may also affect the function of smooth muscle cells. This needs to be further studied. Furthermore, we noticed in the present study that the pressure and stress thresholds to induce contraction increased after obstruction. This can be caused by altered mechanosensory function such as resetting of the mechanoreceptors. This issue also requires further study.

The partially obstructed intestinal model has been validated previously $[10,11,15]$ and demonstrated that it a good model for studying histomorphological and biomechanical intestinal remodeling [14]. The size of the band relative to the circumference of the intestine is important for successful obstruction. If the band is too tight, the animal dies within 48 hours; if it is too loose, no changes will occur [11]. In the present study, the ring was one millimetre bigger than the outer diameter of the intestinal segment. The mortality rate was lower than $20 \%$. Although the band does not compress the resting intestine, during bolus passage the band would increase the resistance during the propagation of contractions by compressing the intestinal wall.

\section{Conclusions}

The total active phasic and tonic stress increased in longterm-obstructed intestine which is likely related to increased total contraction force to push the content through the partial obstruction site. However, the contraction force per smooth muscle unit did not increase in obstructed intestine when compared to normal intestine.

\section{Disclosure}

The authors have no proprietary, financial, professional, or other personal interest related to the research. 


\section{Acknowledgments}

The technicians O. Sørensen, T. Madsen, and J. Sørensen are thanked for handling the animals. This paper was supported by the Aalborg Hospital.

\section{References}

[1] M. Hernanz-Schulman, "Infantile hypertrophic pyloric stenosis," Radiology, vol. 227, no. 2, pp. 319-331, 2003.

[2] M. Miyamoto, K. Egami, S. Maeda et al., "Hirschsprung's disease in adults: report of a case and review of the literature," Journal of Nippon Medical School, vol. 72, no. 2, pp. 113-120, 2005.

[3] W. Park and M. F. Vaezi, "Etiology and pathogenesis of achalasia: the current understanding," American Journal of Gastroenterology, vol. 100, no. 6, pp. 1404-1414, 2005.

[4] J. Cheng, N. Vemula, and S. Gendler, "Small bowel obstruction caused by intramural hemorrhage secondary to anti-coagulant therapy," Acta Gastro-Enterologica Belgica, vol. 71, no. 3, pp. 342-344, 2008.

[5] F. Froehlich, P. Juillerat, C. Mottet et al., "Obstructive fibrostenotic Crohn's disease,” Digestion, vol. 71, no. 1, pp. 29-30, 2005.

[6] T. K. Hsieh, A. C. Chen, S. F. Wu, and W. Chen, "Postoperative intussusception in children with enterostomy," Acta Paediatrica Taiwanica, vol. 46, no. 3, pp. 166-169, 2005.

[7] R. M. Zollinger Jr, W. C. Sternfeld, and H. Schreiber, "Primary neoplasms of the small intestine," American Journal of Surgery, vol. 151, no. 6, pp. 654-658, 1986.

[8] S. Bertoni and G. Gabella, "Hypertrophy of mucosa and serosa in the obstructed intestine of rats," Journal of Anatomy, vol. 199, no. 6, pp. 725-734, 2001.

[9] G. Gabella, "Hypertrophy of visceral smooth muscle," Anatomy and Embryology, vol. 182, no. 5, pp. 409-424, 1990.

[10] K. Schulze-Delrieu, B. Brown, B. Herman et al., "Preservation of peristaltic reflex in hypertrophied ileum of guinea pig," American Journal of Physiology, vol. 269, no. 1, pp. G49-G59, 1995.

[11] J. H. Storkholm, J. Zhao, G. E. Villadsen, H. Hager, S. L. Jensen, and H. Gregersen, "Biomechanical remodeling of the chronically obstructed guinea pig small intestine," Digestive Diseases and Sciences, vol. 52, no. 2, pp. 336-346, 2007.

[12] J. H. Storkholm, J. Zhao, G. E. Villadsen, and H. Gregersen, "Spontaneous and bolus-induced motility in the chronically obstructed guinea-pig small intestine in vitro," Digestive Diseases and Sciences, vol. 53, no. 2, pp. 413-420, 2008.

[13] B. V. Stromberg and L. Klein, "Collagen dynamics of partial small bowel obstruction," American Journal of Surgery, vol. 148, no. 2, pp. 257-261, 1984.

[14] J. Zhao, D. Liao, J. Yang, and H. Gregersen, "Biomechanical remodelling of obstructed guinea pig jejunum," Journal of Biomechanics, vol. 43, no. 7, pp. 1322-1329, 2010.

[15] S. Bertoni, G. Gabella, P. Ghizzardi et al., "Motor responses of rat hypertrophic intestine following chronic obstruction," Neurogastroenterology and Motility, vol. 16, no. 3, pp. 365-374, 2004.

[16] J. Chen, H. Chen, K. M. Sanders, and B. A. Perrino, "Regulation of SRF/CArG-dependent gene transcription during chronic partial obstruction of murine small intestine," Neurogastroenterology and Motility, vol. 20, no. 7, pp. 829-842, 2008.
[17] S. Geuna, S. Cardillo, and M. G. Giacobini-Robecchi, "Smooth muscle cell hypertrophy and hyperplasia in the partially obstructed gut of the rat: a quantitative evaluation," Acta Anatomica, vol. 163, no. 2, pp. 69-74, 1998.

[18] P. A. Longhurst, J. S. Kang, A. J. Wein, and R. M. Levin, "Comparative length-tension relationships of urinary bladder strips from hamsters, rats, guinea-pigs, rabbits and cats," Comparative Biochemistry and Physiology, vol. 96, no. 1, pp. 221-225, 1990.

[19] A. M. Drewes, K. P. Schipper, G. Dimcevski et al., "Multimodal induction and assessment of allodynia and hyperalgesia in the human oesophagus," European Journal of Pain, vol. 7, no. 6, pp. 539-549, 2003.

[20] C. Gao, L. Arendt-Nielsen, W. Liu, P. Petersen, A. M. Drewes, and H. Gregersen, "Sensory and biomechanical responses to ramp-controlled distension of the human duodenum," American Journal of Physiology - Gastrointestinal and Liver Physiology, vol. 284, no. 3, pp. G461-G471, 2003.

[21] H. Gregersen, O. H. Gilja, T. Hausken et al., "Mechanical properties in the human gastric antrum using B-mode ultrasonography and antral distension," American Journal of PhysiologyGastrointestinal and Liver Physiology, vol. 283, no. 2, pp. G368G375, 2002.

[22] J. Zhao, D. Liao, and H. Gregersen, "Phasic and tonic stressstrain data obtained in intact intestinal segment in vitro," Digestive Diseases and Sciences, vol. 53, no. 12, pp. 3145-3151, 2008.

[23] Y. C. Fung, Biomechanics: Motion, Flow, Stress, and Growth, Springer-Verlag, New York, NY, USA, 1990.

[24] H. Gregersen, G. S. Kassab, and Y. C. Fung, "The zerostress state of the gastrointestinal tract: biomechanical and functional implications," Digestive Diseases and Sciences, vol. 45, no. 12, pp. 2271-2281, 2000.

[25] H. Gregersen, Biomechanics of the Gastrointestinal Tract. New Perspectives in Motility Research and Diagnostics, SpringerVerlag, London, UK, 2002.

[26] J. Pedersen, A. M. Drewes, and H. Gregersen, "New analysis for the study of the muscle function in the human oesophagus," Neurogastroenterology and Motility, vol. 17, no. 5, pp. 767-772, 2005.

[27] G. Gabella, "Hypertrophy of intestinal smooth muscle," Cell and Tissue Research, vol. 163, no. 2, pp. 199-214, 1975.

[28] J. Pedersen, C. Gao, H. Egekvist et al., "Pain and biomechanical responses to distention of the duodenum in patients with systemic sclerosis," Gastroenterology, vol. 124, no. 5, pp. 12301239, 2003.

[29] S. Bertoni, V. Ballabeni, L. Flammini, T. Gobbetti, M. Impicciatore, and E. Barocelli, "Intestinal chronic obstruction affects motor responsiveness of rat hypertrophic longitudinal and circular muscles," Neurogastroenterology and Motility, vol. 20, no. 11, pp. 1234-1242, 2008.

[30] G. Gabella, "Hypertrophic smooth muscle. II. Sarcoplasmic reticulum, caveolae and mitochondria," Cell and Tissue Research, vol. 201, no. 1, pp. 79-92, 1979.

[31] G. Gabella, "Hypertrophic smooth muscle. III. Increase in number and size of gap junctions," Cell and Tissue Research, vol. 201, no. 2, pp. 263-276, 1979.

[32] G. Gabella, "Hypertrophic smooth muscle. IV. Myofilaments, intermediate filaments and some mechanical properties," Cell and Tissue Research, vol. 201, no. 2, pp. 277-288, 1979.

[33] D. P. McDaniel, G. A. Shaw, J. T. Elliott et al., "The stiffness of collagen fibrils influences vascular smooth muscle cell phenotype," Biophysical Journal, vol. 92, no. 5, pp. 1759-1769, 2007. 

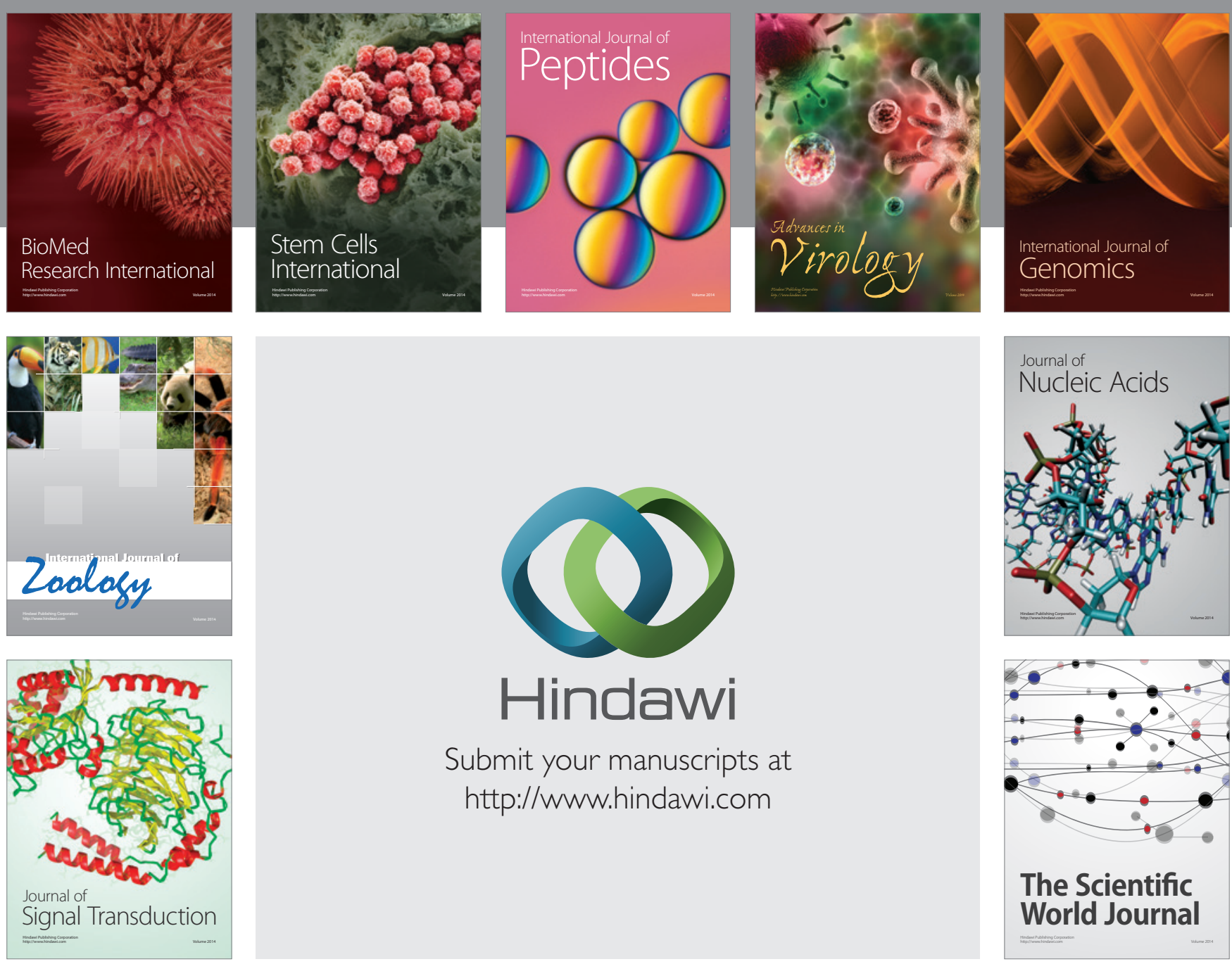

Submit your manuscripts at

http://www.hindawi.com
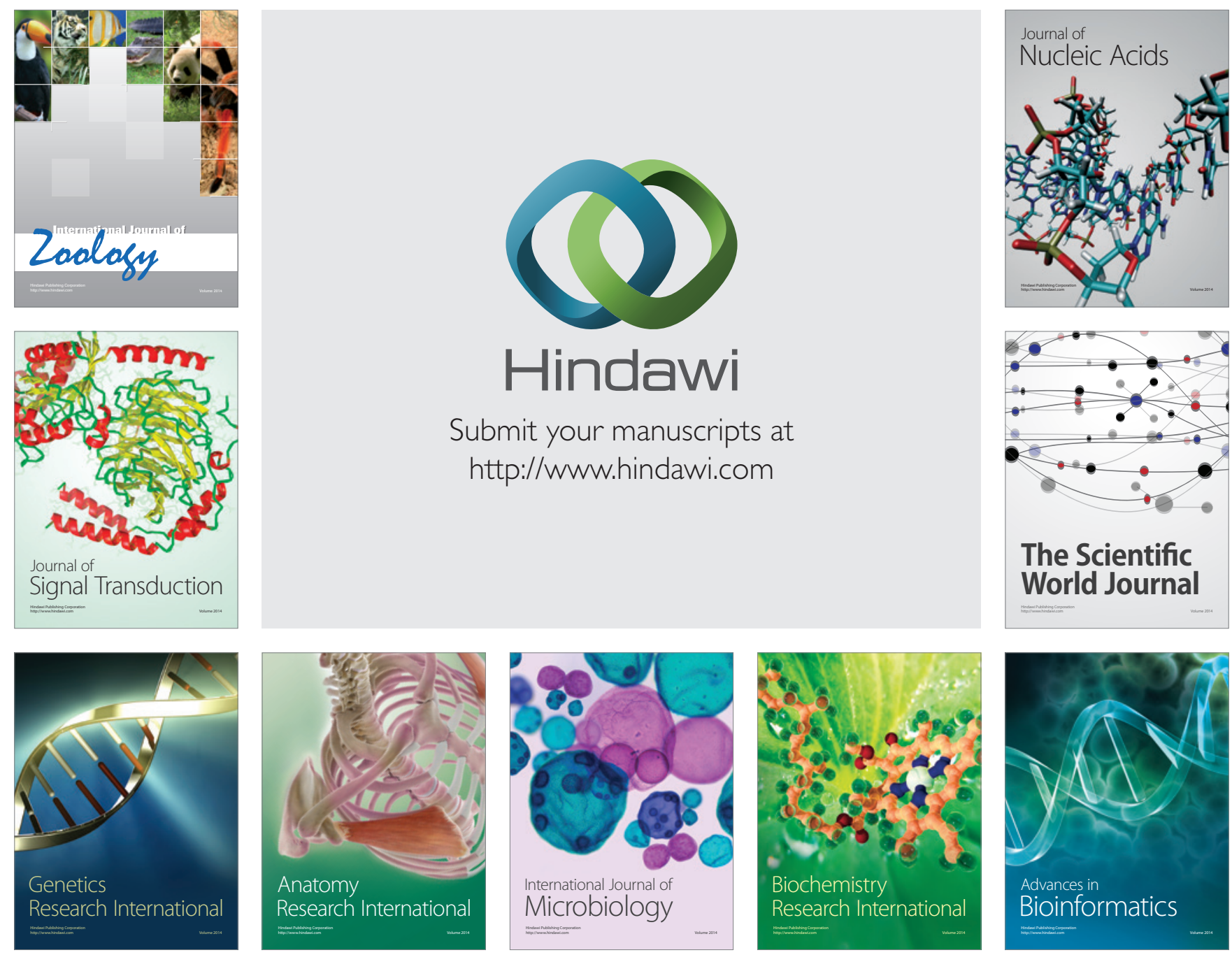

The Scientific World Journal
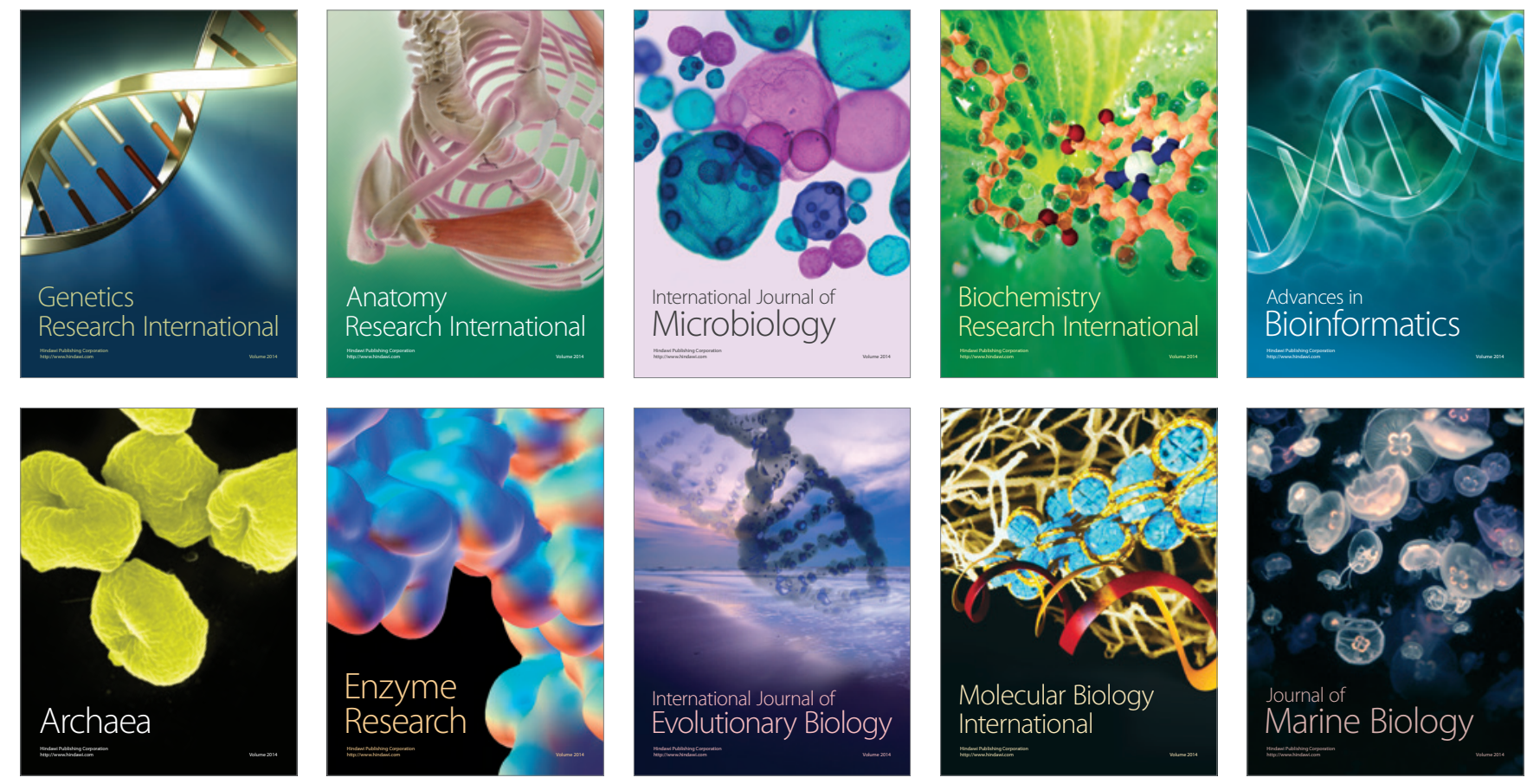